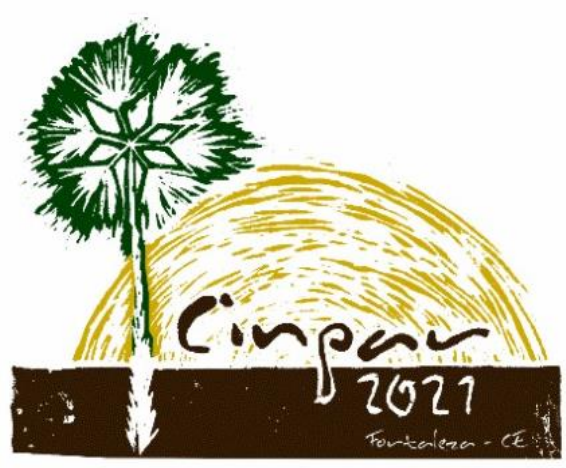

XVII Congresso Internacional sobre Patologia e

Reabilitação das Construções

XVII Congreso Internacional sobre Patología y Rehabilitación de las Construcciones

XVII International Conference on Pathology and Constructions Rehabilitation

FORTALEZA (Brasil), 3 a 5 de junho de 2021

https://doi.org/10.4322/CINPAR.2021.164

\title{
Análisis de ciclo de vida como criterio de elección de los componentes para Pavimentos Drenantes
}

\section{Life-cycle assessment as constituents choice criteria for Draining Pavement}

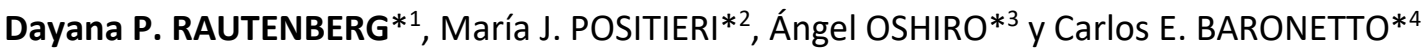 \\ * CINTEMAC (UTN-FRC), Maestro M. López esq. Cruz Roja Argentina - Ciudad Universitaria +54-0351-598-6050/6000 \\ ${ }^{1}$ dayana.rautenberg@gmail.com, ${ }^{2}$ mpositieri@gmail.com, ${ }^{3}$ oshiroangel@gmail.com, ${ }^{4}$ carlosbaronetto@gmail.com
}

Resumen: En muchos países desarrollados, resulta cada vez más habitual el uso de pavimentos drenantes para la construcción de veredas, estacionamientos y entradas de vehículos, pudiendo ser de asfalto u hormigón.

El pavimento drenante se produce utilizando componentes convencionales. Es fundamental una elección sensible de los mismos en su diseño para garantizar durabilidad, satisfacción en su uso y sostenibilidad del proyecto, dada por su ciclo de vida, costo energético de la obtención de materiales, impacto medioambiental de su fabricación, su puesta en obra y su uso durante su vida útil. La elección de los componentes dependerá de las necesidades de respuesta (mecánica e hidráulica), posibilidad de mantenimiento, tecnología de equipos y materiales disponibles en cercanía a la planta de hormigón o asfalto $\mathrm{o}$ al predio del proyecto, y del presupuesto económico asignado al proyecto.

Este artículo presenta experiencias de hormigones drenantes que incluyen la consideración de los conceptos de ciclo de vida para establecer un mecanismo de toma de decisión que pueda adaptarse a los diferentes escenarios constructivos del hormigón drenante y destaca la importancia de la selección de los materiales componentes.

Los estudios de ciclo de vida en pavimentos drenantes aún se encuentran en etapa inicial, aunque ya es posible visibilizar que el ACV es fundamental para orientar la planificación y la toma de decisiones en la elección de los materiales constituyentes.

Palabras claves: pavimento drenante, componentes, ciclo de vida.

Abstract: In many developed countries, it is increasingly common to use draining pavements for sidewalks, parking lots and driveways construction, which it can be made of asphalt or concrete.

Draining pavements is produced using conventional constituents. A sensible choice of these in its design is essential to guarantee durability, satisfaction in its use and sustainability of the project (given by its life cycle, energy cost of obtaining materials, environmental impact of its manufacture, its implementation and its use during its useful life). The components choice will depend on response needs (mechanical and hydraulic), possibility of maintenance, equipment technology and materials available close to the concrete plant or the project site, and the economic budget assigned to the project.

This article presents experiences of draining concrete that include the consideration of life-cycle concepts to establish a decision-making mechanism that can be adapted to the different construction scenarios of draining concrete and highlights the importance of the selection of constituent materials.

Life-cycle studies in draining pavements are still in the initial stage, although it is now possible to see that LCA is essential to guide planning and decision-making in the choice of constituent materials.

Keywords: draining pavement, constituents, life-cycle. 


\section{Análisis del Ciclo de Vida}

Con el aumento de conciencia sobre la previsión y prevención de impactos ambientales relacionados con la construcción, se ha acrecentado el interés en desarrollar métodos para comprender y abordar mejor estos impactos. Una de las técnicas en desarrollo para este propósito es el Análisis del Ciclo de Vida (ACV). El ACV puede identificar oportunidades para mejorar el desempeño ambiental de los productos y servicios en cada fase de su ciclo de vida y establecer indicadores que contribuyan a la toma de decisiones sostenibles en organizaciones gubernamentales o no gubernamentales, por ejemplo: definiendo prioridades y planificando estratégicamente.

El ACV se centra en los posibles impactos ambientales desde la adquisición de las materias primas, producción, uso, tratamiento posterior al uso, reciclaje hasta su disposición final. Los estudios de ACV se componen de cuatro fases: objetivo y alcance; inventario del ciclo de vida; evaluación de impacto; e interpretación (ISO 14040, 2006).

\subsection{ACV de los pavimentos}

La literatura actual demuestra una amplia gama de implicaciones de carga ambiental asociadas con los pavimentos (Santero y Horvath, 2009; Giustozzi, et al., 2012). Chiu et al (2008) demostraron que las acciones dirigidas al desarrollo sostenible en los proyectos de construcción de pavimentos pueden reducir las emisiones de gases de efecto invernadero y su costo de ciclo de vida. Sin embargo, aún existen conceptos sin desarrollar como es el de los pavimentos drenantes. En la literatura se encuentran pocos estudios sobre el ciclo de vida de estos pavimentos y los beneficios ambientales que se pueden lograr mediante la retención de agua y la consecuente reducción de los problemas relacionados con las inundaciones y la recarga hídrica.

Taylor y Patten (2002) han demostrado que los pavimentos de hormigón a base de cemento Portland pueden disminuir la cantidad de combustible consumido en comparación con los pavimentos construidos con asfalto.

Huang et al. (2009) realizaron un ACV sobre un caso de estudio en Reino Unido de una ruta con pavimento asfáltico en el que los agregados naturales fueron reemplazados parcialmente con residuos de vidrio y cenizas de incineración. Los resultados se compararon con un pavimento convencional utilizando únicamente agregados naturales. La mezcla de asfalto, la producción de betún y agregados naturales consumieron, respectivamente, aproximadamente el $62 \%, 23 \%$ y $6 \%$ de la energía total y, en consecuencia, produjeron más emisiones que los otros procesos. El uso de materiales reciclados redujo el consumo de ligante asfáltico en aproximadamente un 7\%, el ahorro de 5.766 toneladas de agregados y el reciclaje de 579 y 989 toneladas de residuos de vidrio y cenizas de incineración, respectivamente. El transporte del agregado natural representó más del 61\% de todo el uso de diésel, debido a la larga distancia de transporte $(193 \mathrm{~km})$. El vidrio y las cenizas se obtuvieron de fuentes locales y el uso de diésel para transportar asfalto fue solo del $17 \%$, ya que el proyecto estaba ubicado muy cerca de la planta de asfalto $(6,4 \mathrm{~km})$.

Los resultados de este estudio muestran la gran dependencia de la ubicación del proyecto vs. los materiales utilizados, los cuales interfieren significativamente con los impactos ambientales del ciclo de vida.

Santero y Horvath (2009) evaluaron el potencial de calentamiento global de los pavimentos convencionales en Estados Unidos, analizando varios componentes como: extracción y producción de materiales, transporte, equipos utilizados, absorción de carbono, islas de calor, rugosidad superficial del pavimento, resistencia a la rodadura, albedo, entre otros. La Figura 1 muestra la emisión de dióxido de carbono (en Mg $\mathrm{CO}_{2}$ equivalente) por kilómetro de ruta durante 50 años. Las barras grises muestran variaciones del potencial de calentamiento global, mientras que las barras negras muestran los valores extremos de cada componente. Los resultados demuestran la amplia gama de posibles impactos a los componentes del ciclo de vida del pavimento. Este impacto varía desde valores insignificantemente hasta $60.000 \mathrm{Mg}$ de $\mathrm{CO} e / \mathrm{km}$ de ruta durante 50 años. 


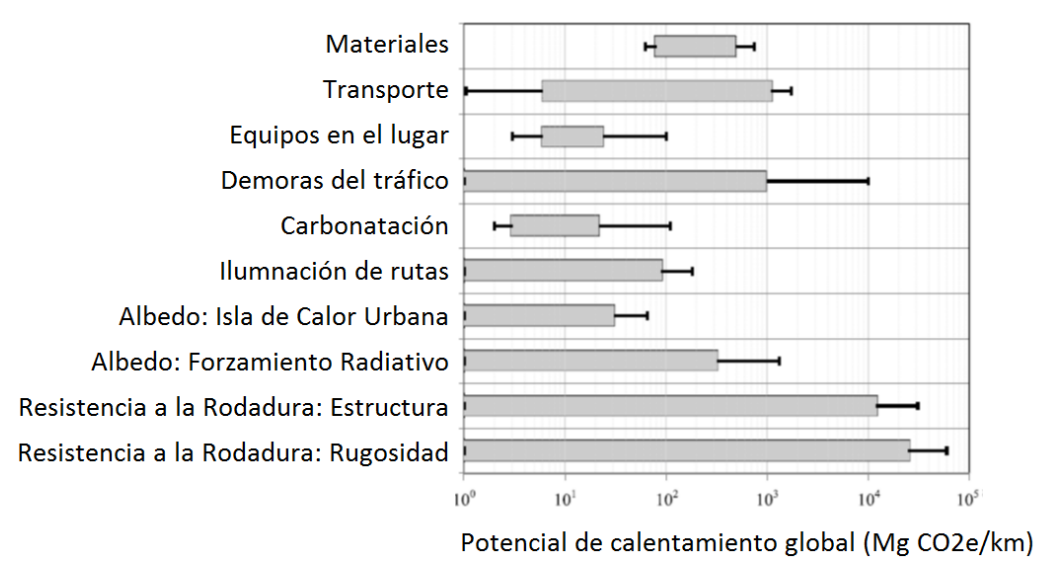

Figura 1 - Impacto del potencial de calentamiento global para los componentes del ciclo de vida del pavimento. Fuente adaptada: Santero y Horvath (2009)

\subsubsection{ACV de los pavimentos drenantes}

En los últimos años, el uso de hormigón drenante como material de pavimentación en aplicaciones viales de bajo tránsito ha ganado importancia debido a sus aspectos ambientales positivos. Debido al mayor uso de hormigón drenante en la industria del pavimento, Chandrappa y Biligiri (2016) afirman que existe un gran margen para futuras investigaciones que lo convertirá en un material prometedor para pavimentos sostenibles en el futuro.

Yuan et al. (2018) compararon los impactos ambientales y económicos de la fabricación de adoquines drenantes ( $10 \%$ de porosidad) con los adoquines convencionales en China. La unidad funcional utilizada en el estudio fue de $1 \mathrm{~m}^{2}$. Todos los insumos de materias primas, consumo de energía, transporte, residuos y descarga de efluentes se calcularon utilizando la unidad funcional y sólo se consideraron las etapas de producción y obtención de materias primas. Los resultados mostraron que el cemento fue el material que causó mayor impacto ambiental en los adoquines drenantes. El resultado del análisis demostró que, si el consumo de cemento se redujera en un $5 \%$, el impacto ambiental general se reduciría en aproximadamente un $2,21 \%$. El coeficiente de permeabilidad de los bloques fue de $1,8 \times 10^{-2} \mathrm{~m} / \mathrm{s}$. Así, durante un período de servicio de 3 años, los adoquines tendrían una capacidad de infiltración de aguas pluviales de 2,01 $\mathrm{m}^{3}$ por $1 \mathrm{~m}^{2}$ de área. Se destaca la importancia de reducir el consumo de cemento.

En definitiva, el estudio del ciclo de vida del hormigón drenante aún cuenta con pocas experiencias en el ámbito internacional y se justifican las experiencias que aporten resultados para avanzar en el conocimiento de este material.

\section{Hormigón drenante}

El hormigón drenante es un material "respetuoso" con el medio ambiente. Se recomienda que se utilice como capa superior de los sistemas de pavimento diseñados para recolectar aguas pluviales en la superficie con el objetivo de evitar inundaciones durante lluvias intensas, recargar el agua subterránea, reducir el efecto de isla de calor urbano y eliminar los contaminantes de la escorrentía de aguas pluviales. Además, el hormigón drenante permite producir hormigón más sostenible y ecológico utilizando materiales reciclados de construcción y demolición (Rahman et al., 2015).

En la Figura 2 se muestra un modelo de la estructura del hormigón drenante. Las partículas del agregado grueso, por lo general de un sólo tamaño, forman el esqueleto de hormigón y la pasta de cemento o el mortero unen dichas partículas (Yang y Jiang, 2003). Esta configuración permite que el agua pueda percolar rápidamente a través del hormigón en detrimento de su resistencia mecánica. Un hormigón drenante típico puede tener un contenido de vacíos que varía de 15 a 30\%, una tasa de percolación de 0,25 a 6,10 mm/s, una densidad de 1.600 a $2.000 \mathrm{~kg} / \mathrm{m}^{3}$ y una resistencia a la compresión de 2 a $28 \mathrm{MPa}$. (ACl 522, 2010) 


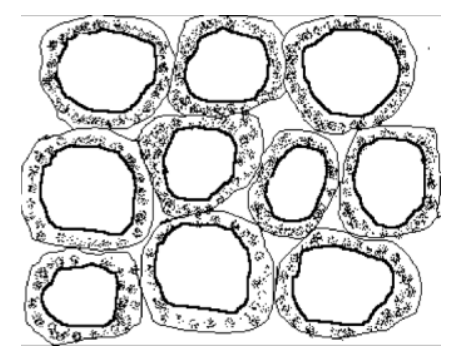

Figura 2 - Modelo esquemático de hormigón drenante (Yang y Jiang, 2003).

Cuando el hormigón se somete a carga, ésta se transfiere a través de la pasta (materiales cementicios y suplementarios + agua + aditivos) entre agregados. La resistencia del agregado es superior a la de la pasta, el espesor de la pasta es muy fino y la interfaz entre los agregados resulta relativamente débil. Por lo tanto, aumentar el espesor/superficie de pasta (sin disminuir los espacios vacíos) y mejorar la resistencia del aglutinante son las claves para mejorar la resistencia del hormigón drenante. El tamaño de las estructuras de los poros (espacios vacíos) y los espesores de la pasta se pueden identificar utilizando un microscopio electrónico de barrido, una tomografía computarizada o una tomografía de rayos X (Torres et al., 2015).

La Tabla 1 proporciona rangos típicos de proporciones de materiales para hormigones drenantes (NRMCA, 2004).

Tabla 1. Valores típicos de proporciones en la dosificación de los hormigones drenantes NRMCA (2004).

\begin{tabular}{|c|c|}
\hline \multicolumn{1}{|c|}{ Material } & Proporciones \\
\hline Material cementicio $(\mathrm{mc})\left[\mathrm{kg} / \mathrm{m}^{3}\right]$ & $270-415$ \\
\hline Agregado (fino Af, grueso $\mathrm{Ag}$ ) $\left[\mathrm{kg} / \mathrm{m}^{3}\right]$ & $1190-1480$ \\
\hline Relación $\mathrm{a} / \mathrm{mc}$, en peso* & $0,27-0,34$ \\
\hline Relación $\mathrm{Ag} / \mathrm{mc}$, en peso* & $4-4,5: 1$ \\
\hline Relación $\mathrm{Af} / \mathrm{Ag}$, en peso** & $0-1: 1$ \\
\hline
\end{tabular}

* Se han usado relaciones más altas, pero reduce significativamente las resistencias y la durabilidad

** La adición de agregado fino disminuirá el contenido vacío y aumentar la resistencia

En los pavimentos drenantes, la estructura del pavimento puede estar compuesta por una combinación de capas, las cuales son: subbase drenante, base drenante, capa de lecho drenante (cuando aplique) y superficie drenante, dimensionada para soportar cargas de tráfico, distribuir tensiones en la subrasante y permitir la filtración del agua (NBR 16416, 2015).

El hormigón drenante se produce utilizando componentes convencionales.

\subsection{Agregados}

Diferentes autores han estudiado y proponen la utilización de agregados redondeados y triturados, tanto normales como livianos, para hacer hormigón drenante. Se debe considerar que cualquiera de ellos debe cumplir con los requisitos de ASTM D448 y C33/C33M (en Argentina: Reglamento CIRSOC 201 y Normas IRAM 1505, 1520 y 1627). El contenido de agregados finos está limitado porque tiende a comprometer la conexión del sistema de poros (volumen de espacios vacíos). La utilización de agregado fino puede aumentar la resistencia a la compresión y la densidad pero como consecuencia, reducir el caudal de agua a través de la masa de hormigón drenante. Sin embargo, hay investigadores que han llegado a la conclusión de que la incorporación de $5 \%$ a $10 \%$ de arena fina, como una relación de masa de agregado fino a agregado grueso, es óptima para mejorar la resistencia sin afectar significativamente la porosidad (lowa State University Report, 2006 y Abou Zeid et al., 2010).

La calidad del agregado en el hormigón drenante es tan importante como en el hormigón convencional. Deben evitarse las partículas alargadas o escamosas, debe estar limpio y libre de recubrimientos, como polvo o arcilla, u otros químicos absorbidos que puedan afectar negativamente la interfase pasta-agregado o la hidratación del material cementicio. La humedad del agregado al momento de mezclar es importante, considerando condición óptima de saturada superficie seca (SSS). De lo contrario, un agregado seco puede resultar en una mezcla que carece de la trabajabilidad para la colocación y compactación y un agregado 
demasiado húmedo pueden contribuir al drenaje de la pasta, causando una obstrucción del volumen de espacios vacíos previsto.

El Instituto Americano del Hormigón ( $\mathrm{ACl}$, American Concrete Institute) sugiere el uso de agregados gruesos de un sólo tamaño o de tamaños entre 19 y $9,5 \mathrm{~mm}$ para hormigones drenantes ( $\mathrm{ACl} 522,2010)$. Sin embargo, algunos investigadores han usado agregados gruesos de menor tamaño entre $9,5 \mathrm{~mm}$ y $2,36 \mathrm{~mm}$ a los fines de aumentar las resistencias mecánicas (Nguyen et al., 2014). Según Crouch et al. (2007) el uso de agregado grueso de menor tamaño da como resultado mayor resistencia a la compresión que el uso de agregado grueso de mayor tamaño y una distribución de porosidad similar. Otra investigación llevada a cabo en la Universidad Estatal de lowa indica que el uso de agregado de un sólo tamaño proporciona una mayor tasa de permeabilidad, pero con una baja resistencia a la compresión del hormigón (Kevern et al., 2014). También se han utilizado agregados de un sólo tamaño de hasta $25 \mathrm{~mm}$. Los agregados más grandes proporcionan una superficie más rugosa, lo que mejora la adherencia neumático-pavimento, la resistencia al deslizamiento y en consecuencia la seguridad. Para pavimentos de poco tráfico y estacionamientos, se prefieren los agregados de menor tamaño ya que su apariencia es más atractiva.

Además del tamaño de los agregados, la mineralogía, textura, forma y distribución granulométrica también influyen en la resistencia mecánica, la permeabilidad y la durabilidad a largo plazo del hormigón drenante. La irregularidad en la forma de los agregados influye en la disposición de las partículas o el modo/equipo de compactación que se deba adoptar. Los agregados de partículas redondeadas requieren menos esfuerzo de compactación que los agregados de partículas angulosas y según experiencias realizadas por Maguesvari y Narasimha (2013) el uso de agregados con partículas angulosas reduce las resistencias mecánicas del hormigón drenante. Sin embargo, las experiencias realizadas en el Centro de Investigación, Desarrollo y Transferencia de Materiales y Calidad (CINTEMAC) en Córdoba, Argentina demuestran que se obtuvieron mejores resistencias en mezclas con agregados de partículas angulosas, tipo "piedra partida", en lugar de redondeadas, tipo "canto rodado", respetando para ambos casos un sólo tamaño de 9,5mm (Rautenberg, 2017).

Según Cosci et al. (2015) la mineralogía del agregado influye más en la distribución de los vacíos que el tamaño de las partículas. Los agregados utilizados son generalmente granito, dolomita, piedra caliza y cuarcita.

\subsection{Materiales cementicios}

Similar al hormigón convencional, en el hormigón drenante es posible utilizar cemento portland Tipo I y II de acuerdo con ASTM C150 y C1157 y cemento portland mezcla que cumplen con ASTM C595 y C1157 (en Argentina Normas IRAM 50000, 500001 y 1624).

\subsubsection{Materiales cementicios suplementarios (MCS)}

Además, se pueden usar materiales cementicios suplementarios (MCS) como cenizas volantes, puzolanas (ASTM C618) y escoria de alto horno granulada molida (ASTM C989) para modificar o mejorar el desempeño del hormigón, el tiempo de fraguado, la tasa de desarrollo de resistencia, permeabilidad, etc.

Lian y Zhuge (2010) informaron que el uso de humo de sílice sin superplastificante puede no ser efectivo para aumentar la resistencia y la razón para este fenómeno es que la inclusión de humo de sílice daría lugar a la floculación de la pasta de cemento y, por lo tanto, intensificar la naturaleza porosa de la mezcla, evitando así el incremento de la resistencia. Las largas moléculas de superplastificante podrían envolver la pasta de cemento provocando la desfloculación y, por lo tanto, mejorando la resistencia.

Khankhaje et al. (2017), en su investigación realizada sobre hormigón drenante sostenible mediante el reemplazo parcial del cemento por cenizas de combustible de aceite de palma, concluyen que el uso de este MCS contribuye a un mayor contenido de vacíos, mayor permeabilidad, menor resistencia a la compresión y tracción que los hormigones drenantes sin MCS. Sin embargo, la resistencia a la tracción y a la compresión obtenidas estuvieron dentro del rango aceptable para rutas de bajo tránsito y estacionamientos. Además, el uso de cenizas de combustible de palma contribuirá a reducir los materiales de desecho de la industria. 
De acuerdo con la revisión de la literatura, no existe una estandarización de las características y porcentajes ideales de MCS que se pueden incorporar a las mezclas de hormigón drenante con el fin de brindar resultados específicos en las resistencias mecánicas y la permeabilidad.

\subsection{Agua}

\section{CIRSOC IRAM calidad}

El agua hidrata el cemento para formar la unión entre las partículas del agregado, generando una mezcla trabajable y proporcionando su resistencia mecánica final. Debido a que el hormigón drenante es muy sensible a los cambios en el contenido de agua, la cantidad precisa de agua es crítica y por lo tanto es necesario realizar ajustes in-situ (CIP 38).

Según NRMCA (2004), la relación a/mc típica se indica en la Tabla 1, presentada anteriormente. Las relaciones $\mathrm{a} / \mathrm{mc}$ más bajas reducen significativamente la trabajabilidad y provocan una cohesión inadecuada debido a la reducción de los enlaces entre las partículas. Un contenido de agua muy bajo también impide el curado adecuado del hormigón y una falla prematura de la superficie por desprendimiento (CIP 38). L relaciones a/c más altas pueden conducir a mezclas menos trabajables que fomentan la segregación y una permeabilidad consecuentemente más baja debido al bloqueo de los huecos. En la Figura 3 se ilustra lo expuesto mediante el ensayo de Han-Squeeze NRMCA, (2004).
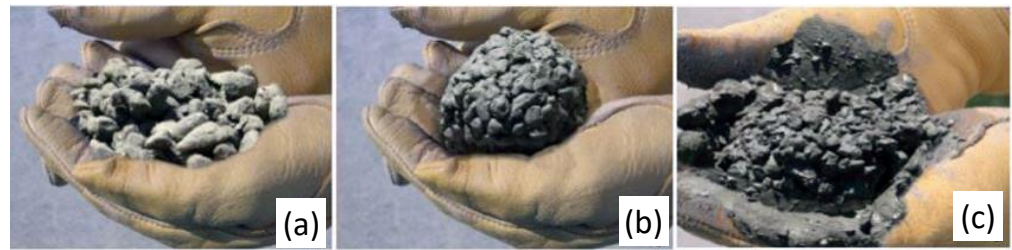

Figura 3. Ensayo de Hand-Squeeze. Muestras de hormigón drenante con diferentes contenidos de agua, formados en una bola: (a) muy poco contenido de agua, (b) cantidad adecuada de agua y (c) demasiado contenido de agua. Fuente adaptada: NRMCA, (2004).

\subsection{Aditivos}

Al igual que en el hormigón convencional, se pueden utilizar aditivos químicos en hormigón drenante para lograr propiedades mejoradas o diferentes. Los retardadores o aditivos estabilizadores de la hidratación se usan comúnmente para superar los problemas asociados con el tiempo de fraguado rápido del hormigón drenante, mientras que un reductor de agua de alto rango siempre se usa para ayudar en la colocación de la mezcla de hormigón drenante actuando como un lubricante entre agregados entrelazados debido a su baja trabajabilidad. Los aditivos incorporadores de aire se utilizan en países con períodos de temperaturas bajo cero, reducen el daño en el hormigón drenante por congelamiento-deshielo. Los aditivos modificadores de la viscosidad son útiles para prevenir el escurrimiento de la pasta (Phillips J., 2009). Los aditivos químicos utilizados deben cumplir con ASTM C 494, mientras que ASTM C 260 rige los aditivos incorporadores de aire.

\subsection{Otros}

También se están usando diferentes agentes poliméricos y microfibras con el fin de aumentar la resistencia y la durabilidad (Wu et al., 2017). Las microfibras son útiles para prevenir el agrietamiento por contracción plástica y pueden mejorar la resistencia a la abrasión y la durabilidad al congelamiento y deshielo. Un estudio realizado por Kevern et al. (2014), especifica la dosis de fibra óptima para procurar permeabilidad y resistencia a la abrasión entre $1,5 \mathrm{~kg} / \mathrm{m}^{3}$ y $3,0 \mathrm{~kg} / \mathrm{m}^{3}$, con longitud de fibra de $56 \mathrm{~mm}$.

\section{Discusiones}

La revisión realizada en este trabajo presenta estudios cuyos resultados demuestran la sostenibilidad que aportan los pavimentos drenantes, considerados como infraestructuras verdes. 
Para estos pavimentos, los estudios de ACV aún presentan heterogeneidad en las unidades funcionales, límites de evaluación, fases, procesos, parámetros y datos mínimos evaluados, entre otros componentes. Por lo tanto, se dificulta comparar los resultados y evaluar los impactos ambientales causados por estos sistemas de drenaje durante sus ciclos de vida.

Se presenta el amplio abanico de materiales constituyentes factibles de usar en hormigones drenantes: agregados gruesos de partículas redondeadas o angulosas, tamaños de partículas que van desde $2,36 \mathrm{~mm}$ a $25 \mathrm{~mm}$, gradación única o fraccionada, agregados naturales o reciclados, distintos contenidos de agregados finos en porcentaje de agregado grueso, cemento portland normal o mezcla reemplazo del cemento por MCS (humo de sílice, ceniza volante, cenizas de combustible de aceite de palma, escoria de alto horno, etc.), incorporación de fibras y aditivos (retardador de fragüe, reductor de agua, incorporadores de aire).

La elección de los constituyentes del hormigón drenante quedará sujeto a los materiales disponibles en la cercanía (o no) al proyecto y del criterio final adoptado por el proyectista justificado por un ACV que abraque desde la obtención de las materias primas hasta su puesta final en obra.

\section{Conclusiones}

Debido al aumento de áreas impermeables y el consecuente aumento de inundaciones en áreas urbanas, la insuficiencia de los sistemas tradicionales de drenaje urbano es cada vez más notoria. La tendencia es que los eventos de inundaciones y otros problemas relacionados con la recarga y contaminación de los recursos hídricos crecerán en los próximos años debido al calentamiento global y los cambios provocados por el hombre. De esta forma, aumenta la importancia de utilizar nuevos sistemas de drenaje sostenibles para mejorar la permeabilidad de las superficies y restaurar el ciclo hidrológico natural. Estos sistemas incluyen a los pavimentos drenantes.

Los estudios de ACV revisados proporcionan una estimación de la sostenibilidad de los pavimentos drenantes. Sin embargo, aún existe la necesidad de una metodología capaz de proporcionar resultados más precisos sobre los impactos ambientales provocados por estos pavimentos. Por lo tanto, la evaluación no debe estar vinculada sólo a los beneficios ambientales relacionados con su vida útil, sino que también son necesarias en los pasos que preceden y siguen a la vida útil.

Si bien los estudios de ciclo de vida en pavimentos drenantes aún se encuentran en etapa inicial, el ACV es una herramienta fundamental para orientar la planificación y la toma de decisiones en la elección de los materiales constituyentes, conduciendo a sistemas que consideren el aumento de los recursos hídricos y la reducción de desastres naturales e impactos ambientales. El hormigón drenante es un material versátil y factible de aplicar en pavimentos independientemente de la ubicación del proyecto, materiales y tecnologías disponibles, reforzando así su concepto y aporte como parte de los sistemas de drenaje urbano sostenible.

\section{Referencias Bibliográficas}

Abou Zeid, Mohamed N., Fahmy, Ezzat H., Kassab, K., Sofy, A., and Saad, M. (2010). Properties and potential of pervious concrete. Proceedings of the CSCE 2nd Structures International Specialty Conference, Winnipeg, Manitoba, Canada.

American Concrete Institute, ACl (2010). ACI 522R-10: Report on Pervious Concrete. American Concrete Institute Committee, Technical Committee Document, Farmington Hills, MI.

Associação Brasileira de Normas Técnicas, ABNT (2015). NBR 16416/2015: Pavimentos Permeáveis de Concreto-Requisitos e Procedimentos. Associação Brasileira de Normas Técnicas (ABNT). São Paulo, Brazil.

Chiu, C.T.; Hsu, T.H.; Yang, W.F. (2008). Life cycle assessment on using recycled materials for rehabilitating asphalt pavements. Resour. Conserv. Recycl., 52, 545-556. 
Chandrappa, A.K.; Biligiri, K.P. (2016). Pervious concrete as a sustainable pavement material-Research findings and future prospects: A state-of-the-art review. Constr. Build. Mater., 111, 262-274.

Cosic, K., et al. (2015). Influence of aggregate type and size on properties of pervious concrete. Construction and Building Materials, 78, 69-76.

Crouch, L.K., Pitt, J. and Hewitt, R. (2007). Aggregate effects on pervious portland cement concrete static modulus of elasticity. Journal of Materials in Civil Engineering, 19, 561-568.

Giustozzi, F.; Crispino, M.; Flintsch, G. (2012). Multi-attribute life cycle assessment of preventive maintenance treatments on road pavements for achieving environmental sustainability. Int. J. LCA, 17, 409-419.

Huang, Y.; Bird, R.; Heidrich, O. (2009). Development of a life cycle assessment tool for construction and maintenance of asphalt pavements. J. Clean. Prod., 17, 283-296.

International Organization for Standardization, ISO (2006). ISO 14040/2006: Environmental ManagementLife-Cycle Assessment-Principles and Framework; International Organization for Standardization (ISO). Geneva, Switzerland.

lowa State University Report (2006). Mix Design Development for Pervious Concrete in Cold Weather Climates. National Concrete Pavement Technology Center, lowa State University Report.

Kevern, J.T., Biddle, D., and Cao, Q. (2014). Effects of macrosynthetic fibres on pervious concrete properties. Journal of Materials in Civil Engineering, 61-6.

Khankhaje E., Hussin M.W., Mirza J., Salim M.R. (2017). Sustainable Pervious Concrete Incorporating Palm Oil Fuel Ash as Cement Replacement. Chemical Engineering Transactions, 56, 445-450.

Lian, C., and Zhuge, Y. (2010). Optimum mix design of enhanced permeable concrete - an experimental investigation. Construction and Building Materials, 24, 2664-2671.

Maguesvari, M.U., and Narasimha, V.L. (2013). Studies on characterization of pervious concrete for pavement applications. Procedia - Social and Behavioral Sciences, 104, 198-207.

National Ready Mixed Concrete Association, NRMCA. (2004). Concrete in Practice: What, Why and How? CIP 38-Pervious Concrete. National Ready Mixed Concrete Association, Silver Spring, MD.

Nguyen, D.H., et al. (2014). A modified method for the design of pervious concrete mix. Construction and Building Materials, 73, 271- 282.

Rahman M.A., Imteaz M.A., Arulrajah A., Piratheepan J., Disfani M.M. (2015). Recycled construction and demolition materials in permeable pavement systems: geotechnical and hydraulic characteristics. Journal of Cleaner Production, 90, 183-194.

Rautenberg, D. P. (2017). Estudio del hormigón permeable como material de construcción en obras viales Contribución a la disminución del Impacto Ambiental asociado a la Urbanización. Tesis de grado Ingeniería Civil, Universidad Tecnológica Nacional, Córdoba, Argentina, 197.

Santero, N.J.; Horvath, A. (2009). Global warming potential of pavements. Environ. Res. Lett., 4, 034011.

Taylor, G.; Patten, J. (2002). Effects of Pavement Structure on Vehicle Fuel Consumption. Centre for Surface Transportation Technology (CSTT): New Delhi, India; National Research Council of Canada (NRC): Ottawa, ON, Canada.

Torres, A., Hu, J., and Ramos, A. (2015). The effect of the cementitious paste thickness on the performance of pervious concrete. Construction and Building Materials, 95, 850-859.

$\mathrm{Wu}, \mathrm{H}$., et al. (2017). Laboratory-simulated investigation on thermal behaviours of permeable concrete pavements. Road Materials and Pavement Design, 18, 97-108.

Yang J., Jiang G. (2003). Experimental study on properties of pervious concrete pavement materials. Cement and Concrete Research, 33, 381-386.

Yuan, X.; Tang, Y.; Li, Y.; Wang, Q.; Zuo, J. (2018). Environmental and economic impacts assessment of concrete pavement brick and permeable brick production process-A case study in China. J. Clean. Prod., 171, 198-208. 